Simulation and laboratory test results of 3D CMS pixel detectors for HL-LHC

This article has been downloaded from IOPscience. Please scroll down to see the full text article.

2012 JINST 7 P08023

(http://iopscience.iop.org/1748-0221/7/08/P08023)

View the table of contents for this issue, or go to the journal homepage for more

Download details:

IP Address: 131.225.23.169

The article was downloaded on 09/08/2013 at 15:03

Please note that terms and conditions apply. 


\title{
Simulation and laboratory test results of 3D CMS pixel detectors for HL-LHC
}

\author{
E. Alagoz, ${ }^{a, 1}$ M. Bubna, ${ }^{a}$ A. Krzywda, ${ }^{a}$ G.F. Dalla Betta, ${ }^{b}$ M. Povoli, ${ }^{b}$ M.M. Obertino, ${ }^{c, e}$ \\ A. Solano, ${ }^{d, e}$ A. Vilela Pereira ${ }^{h}$ K. Arndt, ${ }^{a}$ G. Bolla, ${ }^{a}$ D. Bortoletto, ${ }^{a}$ M. Boscardin, ${ }^{f}$ \\ S. Kwan, ${ }^{g}$ R. Rivera, ${ }^{g}$ I. Shipsey ${ }^{a}$ and L. Uplegger ${ }^{g}$ \\ ${ }^{a}$ Physics Department, Purdue University, \\ West Lafayette, IN 47907-2036, U.S.A. \\ ${ }^{b}$ INFN Padova (Gruppo Collegato di Trento) and Dipartimento di Ingegneria e Scienza dell'Informazione, \\ Università di Trento, \\ Via Sommarive 14, I-38123 Povo di Trento (TN), Italy \\ ${ }^{c}$ Università del Piemonte Orientale, \\ Novara, Italy \\ ${ }^{d}$ Università di Torino, \\ Torino, Italy \\ ${ }^{e}$ INFN, Sezione di Torino, \\ Torino, Italy \\ ${ }^{f}$ Centro per i Materiali e i Microsistemi Fondazione Bruno Kessler (FBK), \\ Trento, Via Sommarive 18, I-38123 Povo di Trento (TN), Italy \\ ${ }^{g}$ Fermilab, \\ Batavia, IL 60510-5011, U.S.A. \\ ${ }^{h}$ Instituto de Física, Universidade do Estado do Rio de Janeiro (UERJ), \\ Rua São Francisco Xavier, 524, 20550-013 Rio de Janeiro, RJ, Brazil \\ E-mail: enver.alagoz@cern.ch
}

\footnotetext{
${ }^{1}$ Corresponding author.
} 
ABSTRACT: The CMS pixel detector is the innermost tracking device at the LHC, reconstructing interaction vertices and charged particle trajectories. The current planar sensors located in the innermost layer of the pixel detector will be exposed to very high fluences which will degrade their performances. As a possible replacement for planar pixel sensors in the High Luminosity-LHC (HL-LHC), 3D silicon technology is under consideration due to its expected good performance in harsh radiation environments. Studies are also in progress for using 3D silicon pixel detectors in near-beam proton spectrometers at the LHC. Deep Reactive Ion Etching (DRIE) plays a key role in fabricating 3D silicon detectors in which readout and ohmic electrodes are processed through the silicon substrate instead of being implanted on the silicon surface. 3D pixel devices considered in this study were processed at FBK (Trento, Italy), bump bonded to the CMS pixel readout chip, and characterized in the laboratory. Numerical simulations were also carried out. We report on selected results from laboratory measurements and TCAD simulations.

KEYWORDS: Large detector systems for particle and astroparticle physics; Particle tracking detectors (Solid-state detectors) 


\section{Contents}

1 Introduction 1

2 Sensors 2

3 Simulation $\quad 4$

4 Laboratory measurements 9

4.1 Sample preparation 9

4.2 Experimental setup 9

5 Results 10

$\begin{array}{ll}5.1 \text { Leakage current } & 10\end{array}$

5.2 Noise 11

5.3 Charge collection 13

6 Comparison of simulation results with laboratory measurements $\quad 14$

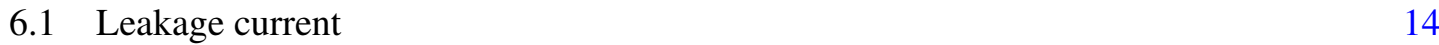

6.2 Charge collection 15

6.3 Noise 16

7 Conclusions 17

\section{Introduction}

The Large Hadron Collider (LHC) at CERN will be upgraded to peak luminosity of $5 \times 10^{34}$ $\mathrm{cm}^{-2} \mathrm{~s}^{-1}$ in 2020 or later to reach its physics goals. At this luminosity upgrade, known as High Luminosity-LHC or HL-LHC, the CMS innermost silicon tracking devices will need to perform reliably in a very harsh radiation environment. These tracking devices will be exposed to fluences on the order of $10^{16} 1 \mathrm{MeV}$ neutron equivalent per $\mathrm{cm}^{2}\left(\mathrm{n}_{\mathrm{eq}} / \mathrm{cm}^{2}\right)$ at HL-LHC luminosity [1]. Since the current CMS pixel tracker, which has standard planar silicon sensors, cannot withstand such high fluences, new studies are ongoing to find radiation hard technologies to replace the current detectors $[2,3]$. Studies are also in progress for using three-dimensional (3D) silicon pixel detectors in near-beam proton spectrometers at the LHC [4].

The 3D architecture for radiation hard silicon detectors using electrodes that pass through the entire silicon substrate was first introduced in 1997 [5]. This new technology decouples the electrode distance from the active substrate thickness providing several superior features, which makes it more radiation hard, compared to planar technology: (i) low depletion voltages and high electric fields between the electrodes, (ii) fast charge collection due to high average electric field, (iii) shorter inter-electrode or charge collection distances and consequently low charge trapping 
probability, and therefore good charge collection efficiency. The first full 3D devices were fabricated with fully penetrating electrodes at Stanford [6]. Later, the fabrication process was also developed at SINTEF (Oslo, Norway) for larger production as a part of 3D collaboration [7, 8]. A modified-3D device approach, where readout and bias electrodes do not pass through the entire substrate thickness and overlap partially, emerged to simplify the fabrication process of 3D devices as opposed to full-3D approach $[9,10]$. An additional feature developed with the 3D architecture is the possibility to reduce the dead area at the edges by creating an "active-edge", i.e. a deep trench all around the detector allowing full collection efficiency a few microns away from the device's physical edge [11-13]. As a matter of fact, the conventional planar devices can also be fabricated with an active edge in place of guard rings, but they are not suitable for high radiation environments $[14,15]$. These aforementioned advantages make 3D sensors a very strong candidate to replace the current planar silicon sensors in the CMS pixel detector. However, 3D detectors present some challenges: (i) the Deep Reactive Ion Etching (DRIE) introduces some complications in the fabrication process making 3D technology more expensive than standard planar technologies, (ii) the configuration of the electrodes causes the pixel capacitance to increase, with possible impact on the noise at the output of the read-out chain, (iii) some low-field regions are present between electrodes of the same doping type causing some parts of the devices to be slightly less efficient in collecting charge.

This paper reports laboratory measurements and simulation results for the CMS 3D silicon sensors designed at the University of Trento and processed at Fondazione Bruno Kessler (FBK), Trento, Italy.

\section{Sensors}

The 3D sensors considered in this study are the Double side Double Type Column (3D-DDTC). These devices are the result of studies conducted independently both at FBK [9] and CNMIBM [10] aimed at the simplification of the fabrication process of 3D silicon radiation detectors. The 3D-DDTC process is a double-sided process, with junction electrodes etched from the front side and ohmic electrodes etched from the back side of the wafer. In the first generation of 3D-DDTC devices fabricated at FBK, columnar electrodes did not pass through the entire silicon bulk, resulting in the presence of additional low-field regions between the tip of the columns and the opposite wafer surface [9]. Moreover, the calibration of the DRIE step to obtain the desired column depth is not an easy task, and non-uniformities in the electrode overlap can affect the device performance.

The following generation of FBK 3D-DDTC sensors, which is the object of this study, is a direct evolution of the previous one. Devices were fabricated in Float Zone p-type high-resistivity wafers with a nominal thickness of $200 \pm 20 \mu \mathrm{m} ; \mathrm{n}^{+}$(readout) electrodes were etched from the front side while $\mathrm{p}^{+}$(ohmic) electrodes were etched from the back side. Both electrode types completely pass through the silicon bulk and are hollow, and metal contact is made on the wafer surface by small planar diffusion. Surface isolation between readout electrodes is realized by means of $\mathrm{p}$ spray implantations on both wafer sides. A schematic cross-section of a 3D-DDTC sensor with pass through electrodes is shown in figure 1. More details about this specific architecture can be found in $[16,17]$. 


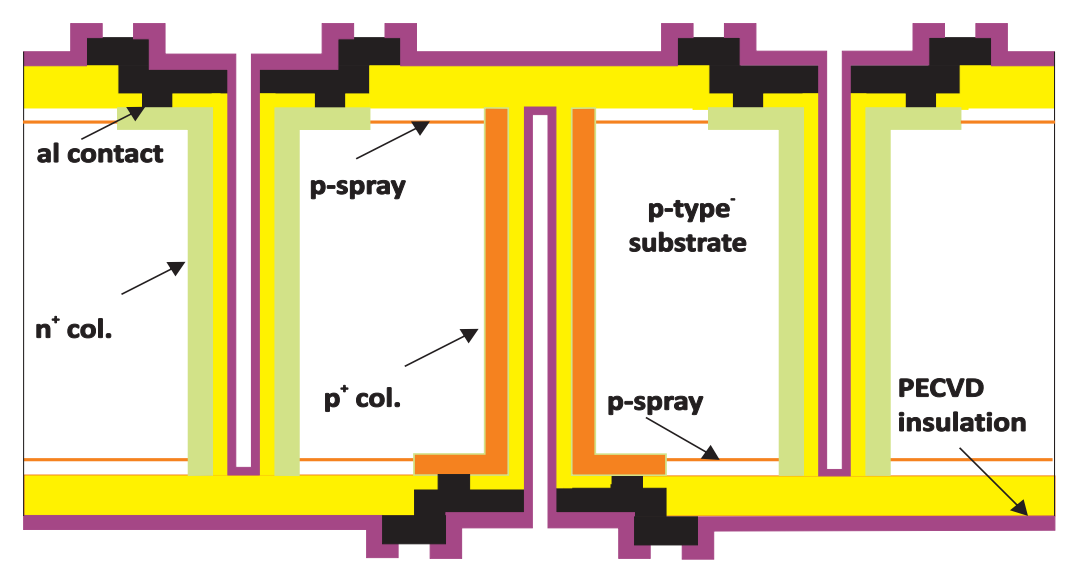

Figure 1. Schematic cross-section of a 3D-DDTC sensor with electrodes penetrating into a p-type substrate (not to scale).

As these devices are double-sided, it is not possible to use a support wafer, which would make the backside of the sensor wafer inaccessible. For this reason 3D-DDTC detectors do not have an active-edge. The devices considered in this paper have a standard edge region about $1 \mathrm{~mm}$ wide, with several planar guard-rings surrounding the active area. This has been significantly improved in later batches at FBK by implementing the so-called "slim-edge", with a dead region of $200 \mu \mathrm{m}$ or below [18].

Figure 2 shows the wafer layout of the 3D-DDTC sensors under study. Only about $30 \%$ of the wafer is devoted to CMS 3D sensors. The remaining wafer area is covered with the ATLAS 3D sensors and other test structures. This paper reports on the results of CMS 3D sensors from two wafers processed in 2010 in the batch called ATLAS08.

Three different pixel configurations were designed and manufactured, each of them compatible with the existing CMS pixel readout chip (ROC) type PSI46v2 [19], which has an array of 80 rows $\times 52$ columns of $100 \mu \mathrm{m} \times 150 \mu \mathrm{m}$ readout pixels. The different pixel configurations are: (i) single n-type electrode (1E), (ii) two n-type electrodes (2E), and (iii) four n-type electrodes (4E) per pixel cell as shown in figure 3 . The distance between n-type and p-type electrodes is of great importance since it affects parameters such as capacitance and noise, depletion voltage, charge collection and radiation hardness. The inter-electrode distances in the 1E, $2 \mathrm{E}$, and $4 \mathrm{E}$ configurations are $90 \mu \mathrm{m}$, $62.5 \mu \mathrm{m}$, and $45 \mu \mathrm{m}$, respectively. For the $1 \mathrm{E}$ configuration, the inter-electrode distance refers to the ohmic electrodes along the diagonal directions, but it should be noted that two additional ohmic electrodes are present along the vertical direction at $50 \mu \mathrm{m}$ from the $\mathrm{n}+$ electrode, reducing low field regions between $n+$ electrodes. Readout columns (n-type) are shorted together inside each pixel. A single $\mathrm{n}^{+}$column is surrounded by six $\mathrm{p}^{+}$columns in the $1 \mathrm{E}$ configuration and by four $\mathrm{p}^{+}$columns in the $2 \mathrm{E}$ and $4 \mathrm{E}$ configurations. Figure 4 shows a detail of a $1 \mathrm{E}$ sensor. It is important to notice that both types of electrodes are visible, indicating that all the holes completely pass through the silicon wafer. A slight misalignment is present between columns of opposite doping type. This effect is caused by the wafer bowing which was quite large in this batch, making alignment challenging. Wafer bowing was reduced and alignment improved in later batches [17]. 


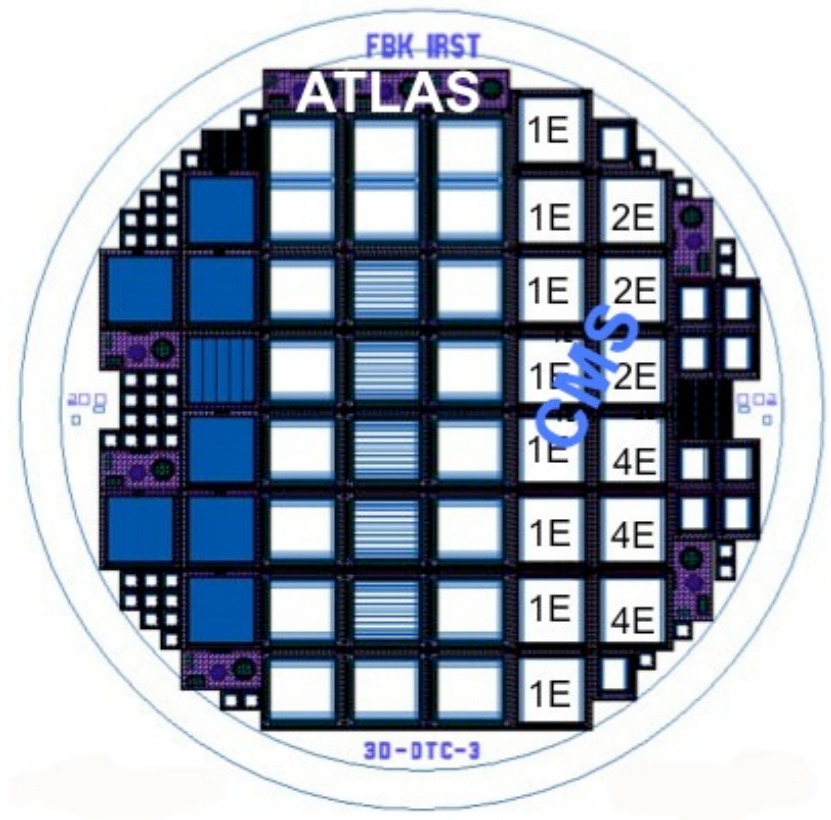

Figure 2. Wafer layout of 3D-DDTC sensors fabricated at FBK (batch ATLAS08, 2010).

\section{Simulation}

A thorough simulation was made with the Synopsys TCAD Tools [20] to fully understand 3DDDTC sensor characteristics and performances. Simulations are carried out by solving continuity and Poisson's equations simultaneously, including carrier drift, diffusion, generation, and recombination according to the Shockly-Read-Hall statistics and avalanche generation.

The complexity and the time needed to perform the required simulation was significantly reduced by selecting a small section of the pixel cell and by using the symmetry of a single pixel. Simulation results were then scaled to the full size of the simulated device. The simulation domains are shown in figure 3. Throughout the simulations a substrate thickness of $200 \mu \mathrm{m}$ and an electrode diameter of $12 \mu \mathrm{m}$ were used. The simulated pixel cell structure of the $4 \mathrm{E}$ sensor configuration is shown in figure 5. The substrate is p-type with a doping concentration of $7 \times 10^{11} \mathrm{~cm}^{-3}$ corresponding to a resistivity of $\sim 20 \mathrm{k} \Omega \cdot \mathrm{cm}$. The doping concentration of ohmic $(\mathrm{p}+)$ and readout $(\mathrm{n}+)$ electrodes is assumed to be $5 \times 10^{19} \mathrm{~cm}^{-3}$. All parameters are representative of FBK 3D technology.

Figure 6 shows the simulated leakage current for all electrode configurations. All investigated devices exhibit a full depletion voltage lower than $10 \mathrm{~V}$, as evident from the early knee in the I-V curves. The different spacing of the $\mathrm{n}^{+}$electrodes affects the shape of the I-V curves before full depletion: $4 \mathrm{E}$ and $1 \mathrm{E}$ configurations are the first and the last respectively reaching the plateau. This is due to the different inter-electrode distances of the various configurations (higher interelectrode distance translates into higher full depletion voltage). The simulated leakage current at full depletion is less than $20 \mathrm{nA}$ for all devices. Breakdown voltages are $33 \mathrm{~V}, 31 \mathrm{~V}$, and $30 \mathrm{~V}$ for $1 \mathrm{E}, 2 \mathrm{E}$, and $4 \mathrm{E}$ respectively. The breakdown behavior of these $\mathrm{p}$-spray isolated $3 \mathrm{D}$ devices was found to depend on the top surface layout rather than the inter-electrode distance. In particular, the critical region seems to be the curvature of the $\mathrm{n}^{+}$planar diffusion to $\mathrm{p}$-spray junction on the front 


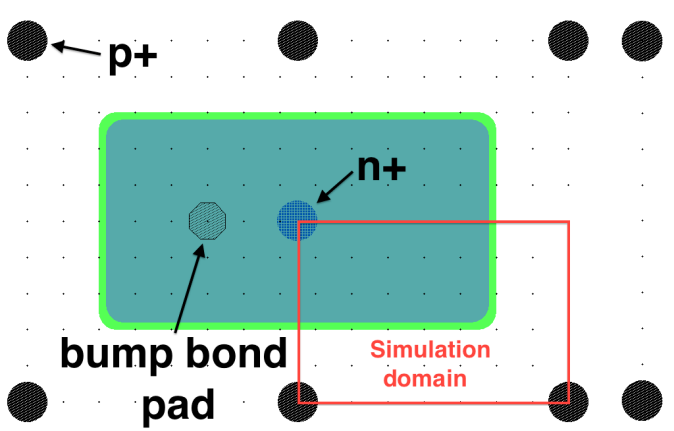

(a)

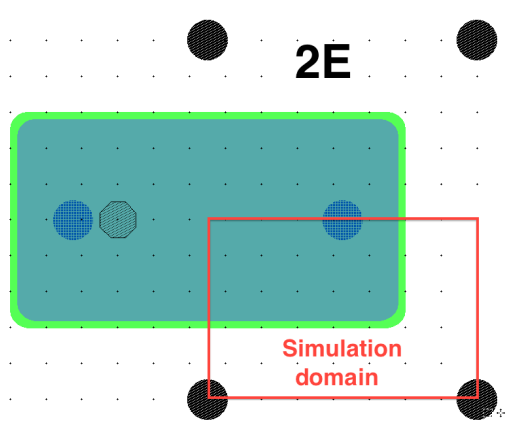

(b)

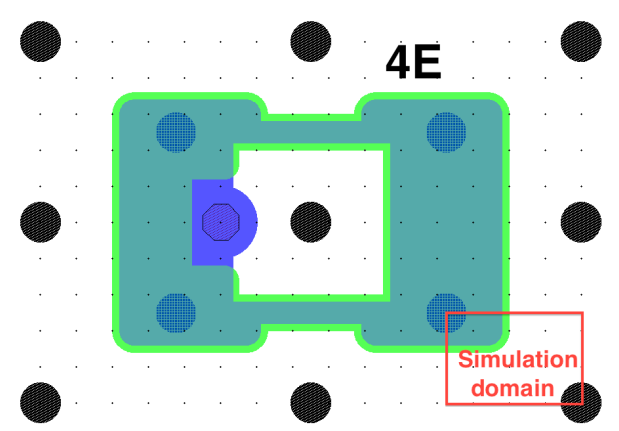

(c)

Figure 3. Sketch of the different pixel configuration, (a) 1E, (b) $2 \mathrm{E}$ and (c) 4E. Simulation domains are highlighted in red.

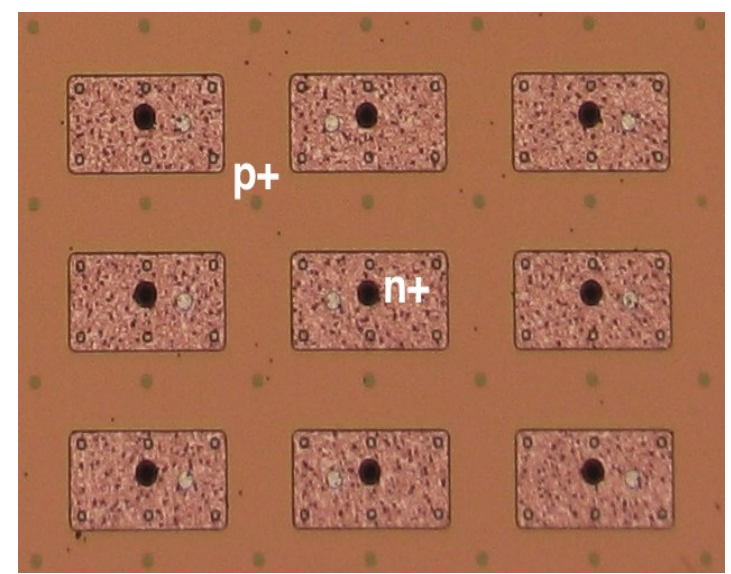

Figure 4. Microscope image of the CMS 3D pixel detector in the 1E configuration. Both electrode types are visible and it is possible to notice a slight misalignment between them.

side. This effect is amplified by the fact that, in 3D detectors with pass through electrodes, the bias columns $\left(\mathrm{p}^{+}\right)$conduct the same voltage applied to the back side directly to the p-spray on the front side. More details can be found in [21]. 

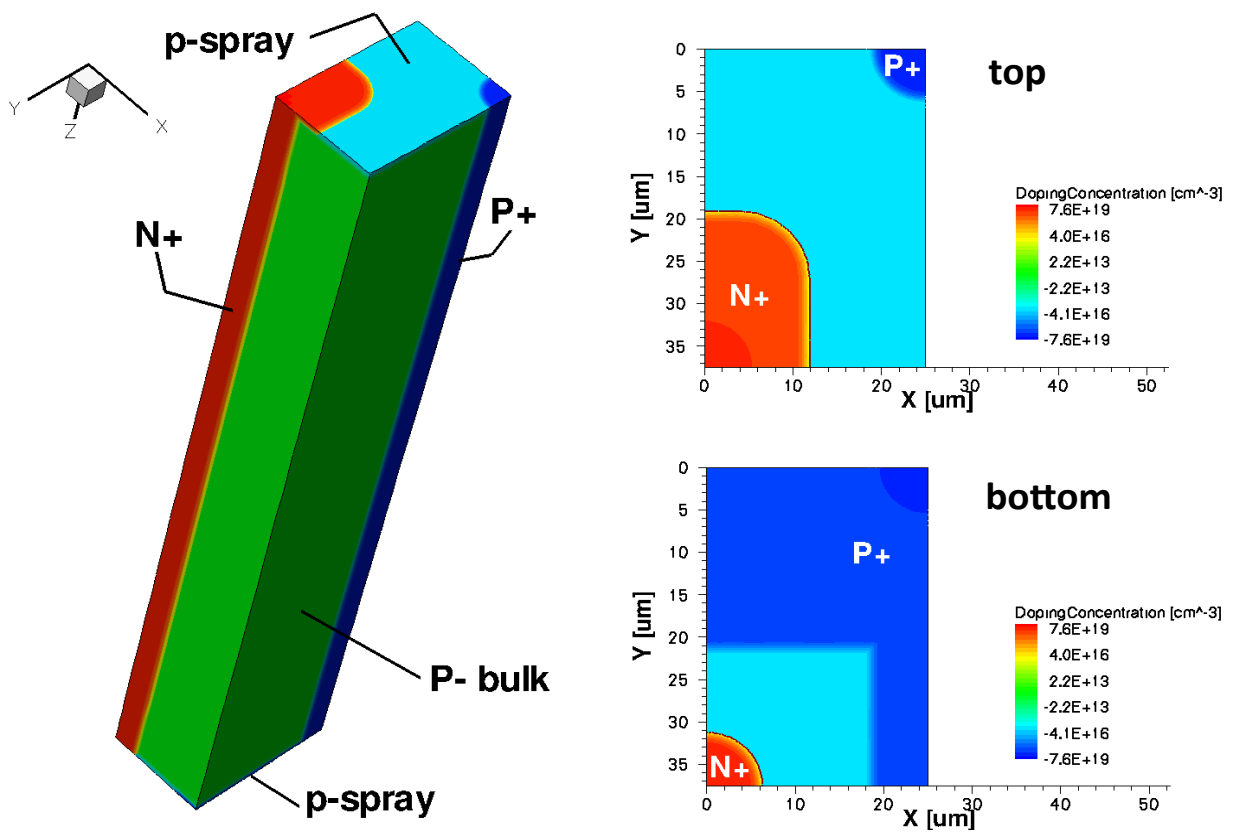

Figure 5. Simulated pixel cell structure of a 4E configuration.

Capacitance versus voltage simulations are shown in figure 7. In all three configurations the capacitance decreases as a function of the bias voltage. The curve tends to stabilize for bias voltages of at most $10 \mathrm{~V}$, confirming that the expected full depletion voltage should be lower than $10 \mathrm{~V}$. It is important to notice that, although the capacitance becomes almost constant after full depletion in all configurations, there still is a slight slope in the curves that can be attributed to the presence of an additional capacitance component related to the $\mathrm{n}^{+}$to $\mathrm{p}$-spray junctions. As voltage increases it is possible to also partially deplete a small part of the p-spray on both wafer sides, thus slightly reducing the capacitance of the device. This behavior is also confirmed by the $1 / C^{2}$ curves shown in the inset of figure 7 (note that the kinks in the curves are due to a mesh effect in the simulation). As for the trend in the capacitance values, simulated results are in agreement with the expectations, as the capacitance properly scales with the number of $\mathrm{n}^{+}$columns per pixel.

The charge collection simulation has been carried out with a transient technique. A Minimum Ionizing Particle (MIP) produces in average $\sim 80$ electron-hole pairs per micron, when it traverses the silicon substrate. In addition, the most probable value corresponds to $\sim 75$ electron-hole pairs per micron. The spatial distribution of generated electron-hole pairs is assumed to be Gaussian within a one micron radius around the particle track. The transient simulation is performed from 0 to $100 \mathrm{~ns}$ for two locations in each electrode configuration. In figure 8 , simulated particle hit points in the domain cell for the different electrode configurations are shown. The first location is at the center and the second is at the corner of the simulation domain to account for different electric field conditions. The simulated current pulse is then numerically integrated and the value of the collected charge is extracted.

In figure 9, simulated charge collection time as a function of reverse bias voltage for the 


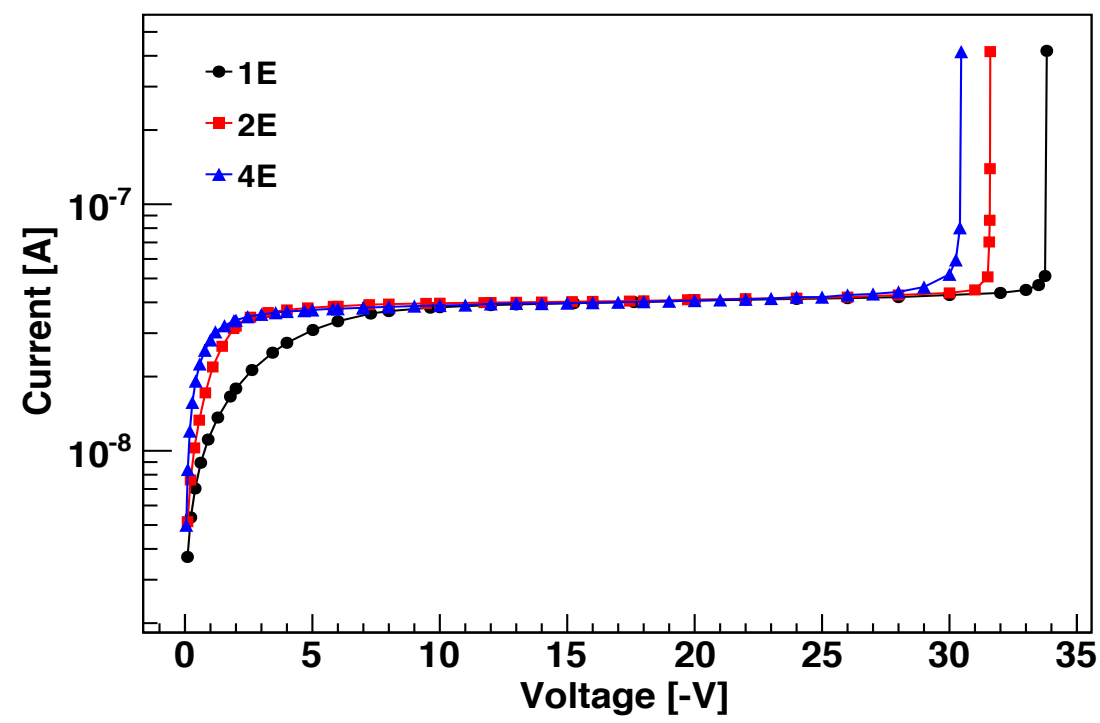

Figure 6. Simulated leakage current for the different electrode configurations.

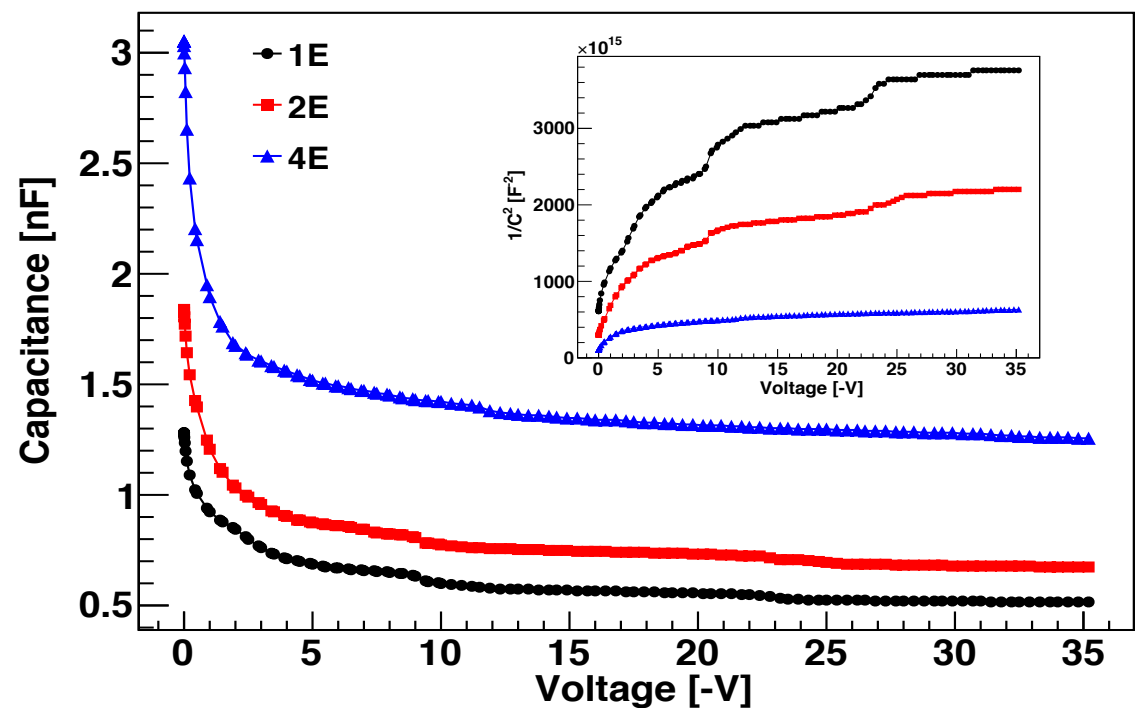

Figure 7. Simulated capacitance for the different electrode configurations. The corresponding $1 / \mathrm{C}^{2}$ versus reverse bias is shown in the inset.

different electrode configurations is shown. Each time corresponds to a full collection of the charge expected from a MIP. A shorter electron drift distance corresponds to a faster charge collection. At a reverse bias voltage of $30 \mathrm{~V}$, for a MIP hitting at the center of the cell, the collection time is only $1 \mathrm{~ns}$ in the $4 \mathrm{E}$ configuration, whereas it increases to almost $10 \mathrm{~ns}$ in the $1 \mathrm{E}$ configuration. Higher charge collection times occur when the particle hits near the corner of the cell. The $1 \mathrm{E}$ device exhibits the worst performance with a time of about $20 \mathrm{~ns}$ at $30 \mathrm{~V}$ bias.

Simulated values of the collected charge after $20 \mathrm{~ns}$ as a function of reverse bias voltage for 
CMS 1E

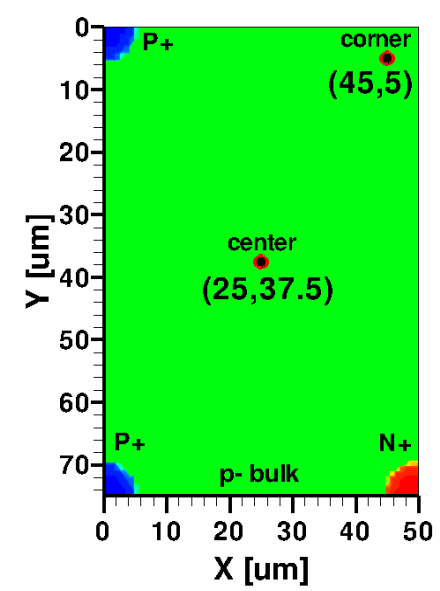

CMS 2E

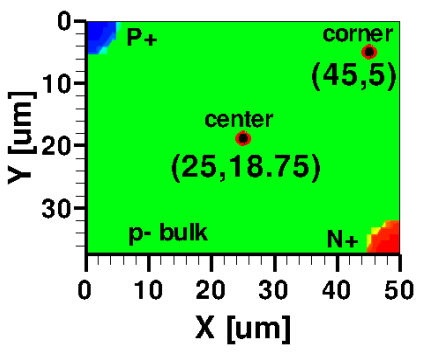

Doping Conc. [ $\left.\mathrm{cm}^{\wedge}-3\right]$

$5.0 \mathrm{E}+19$

$3.2 E+16$

$2.0 \mathrm{E}+13$

$-2.0 \mathrm{E}+13$

$-3.2 E+16$

$-5.0 \mathrm{E}+19$

Figure 8. Simulated particle hit points in the domain cell for the different electrode configurations.

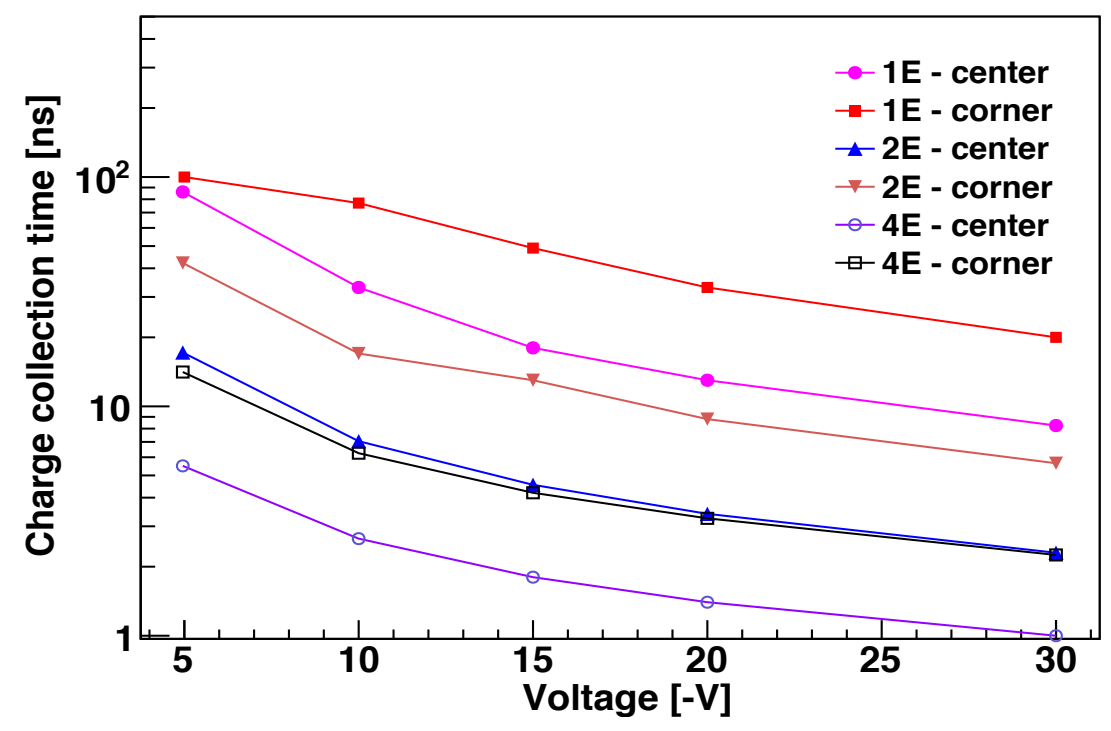

Figure 9. Simulated charge collection time as a function of reverse bias voltage for the different electrode configurations.

all electrode configurations are shown in figure 10. For MIPs hitting the center of the cells, sensors of all electrode configurations have a charge collection plateau at $16 \mathrm{k} e^{-}$which corresponds to the value expected for a MIP traversing a $200 \mu \mathrm{m}$ thick silicon substrate. For the $1 \mathrm{E}$ configuration, the plateau is reached at $10 \mathrm{~V}$ bias voltage. For MIPs hitting at the corners in $1 \mathrm{E}$ and $2 \mathrm{E}$ sensors, the plateau is reached at higher voltages. This is due to the lower electric field in these regions causing 


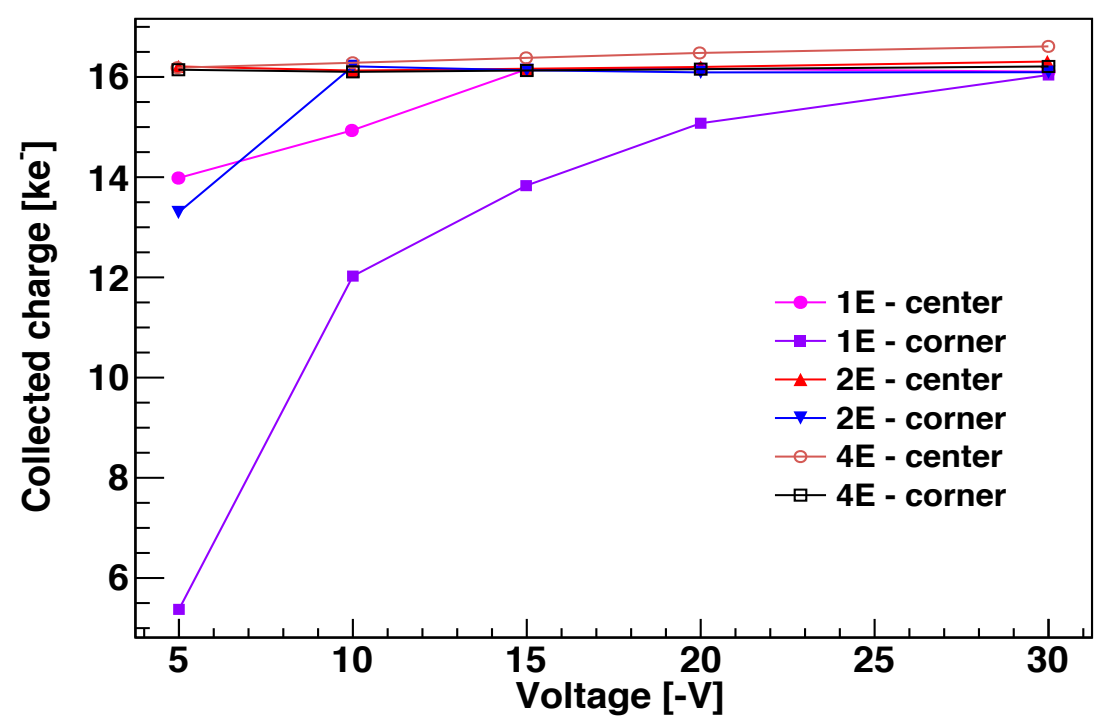

Figure 10. Simulated values of the collected charge after $20 \mathrm{~ns}$ are shown as a function of reverse bias voltage for the different electrode configurations.

longer drift times as seen in figure 9 , whereas, in the corner of a 4E, the full charge collection takes place at very low voltages.

\section{Laboratory measurements}

\subsection{Sample preparation}

FBK sensors were diced and bump bonded to the CMS PSI46v2 ROC at SELEX Sistemi Integrati, Italy. The bump-bond solder material is indium. Half of the bump-bonded sensors were assembled in a clean room in the Purdue Particle Physics Microstructure Development Laboratory (P3MD) at Purdue University. The assembly procedure is similar to that of the production of CMS forward pixel detector modules, which are currently operating in the CMS tracker, as described in [22]. Bump-bonded ROCs were glued and wire bonded to a Very High Density Interconnect (VHDI) circuit. The VHDI was then wire bonded to a fan-out connection board. The fan-out board and VHDI were glued to a carbon fiber base plate, completing the assembly. A bias wire board was used between the sensor back plane and the corresponding wire bond pad on the fan-out board for the high voltage connection to the sensor. A picture and an artistic sketch of the 3D detector are shown in figure 11.

The other half of the bump-bonded sensors were assembled at the INFN Torino. Single detectors are glued on single-chip testboards, specifically designed for the testbeam. Then ROCs are wire bonded to the testboards.

\subsection{Experimental setup}

Laboratory characterization measurements of 3D detectors are performed in the P3MD lab and INFN laboratories. The experimental setup for readout characterization and radioactive source testing of 3D detectors consists of a DAQ board, a 3D detector adapter, and a PC. The DAQ board 


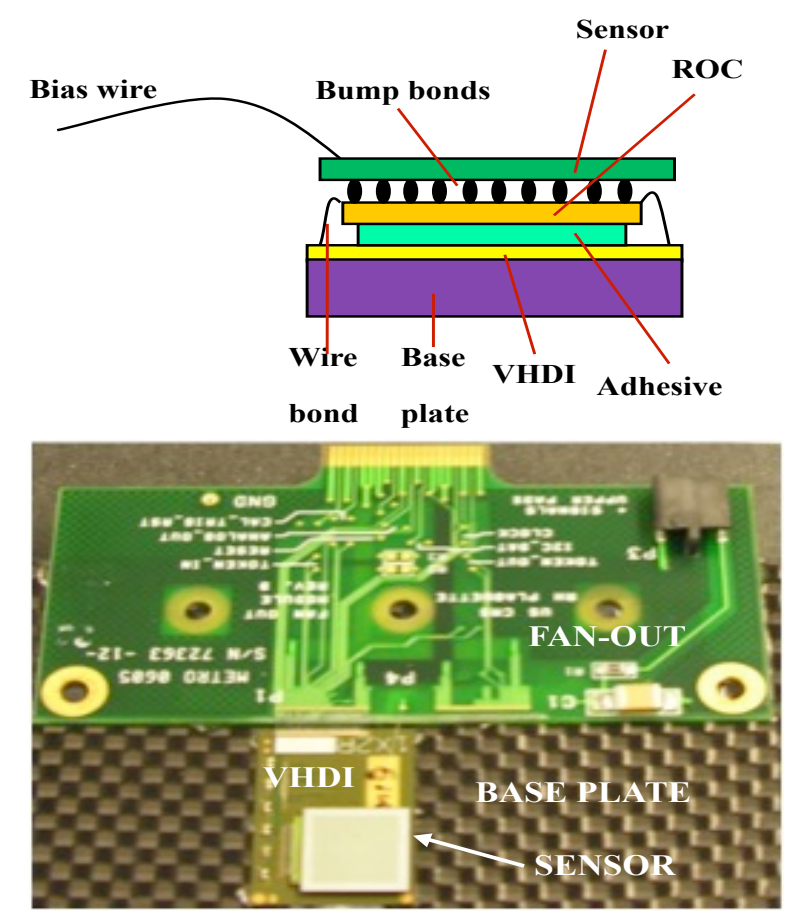

Figure 11. A photo and an artistic sketch of the 3D sensor assembly.

has a Field Programmable Gate Array (FPGA), a 12-bit analog-to-digital converter (ADC), and a $64 \mathrm{MB}$ SDRAM buffer. The setup is connected to a PC via USB serial communication. The DAQ board and ROC characterization software were developed to perform module qualifications measurements for the current CMS barrel pixel detector [23]. An Agilent E3631A model power supply is used to provide $6 \mathrm{~V}$ to operate the DAQ board. A Keithley 2410 model source-meter is used to bias the sensors and to measure the leakage current.

Charge collection measurements are performed by using a $1 \mathrm{mCi}{ }^{90} \mathrm{Sr}$ radioactive source that emits electrons with peak energy of $0.5 \mathrm{MeV}$. The source has a $50 \mu \mathrm{m}$ opening and it was placed $1 \mathrm{~cm}$ away from the detector under test. A random (self) trigger is used during the 3D detector charge collection measurements. Only half of the assembled sensors are tested with the source.

\section{Results}

Several FBK 3D-DDTC sensors with 1E, 2E, and 4E configurations were tested in the laboratories. All measurements were carried out at room temperature. Results are presented in the following sections. In all figures, detectors are labeled to indicate the electrode configuration, the sensor number, and the wafer number respectively.

\subsection{Leakage current}

Leakage current measurements are carried out by applying reverse bias voltages with the Keithley 2410 source-meter. The procedure is controlled by a PC via National Instrument LabVIEW software. At each reverse bias voltage, currents are averaged over a few readings in order to avoid 


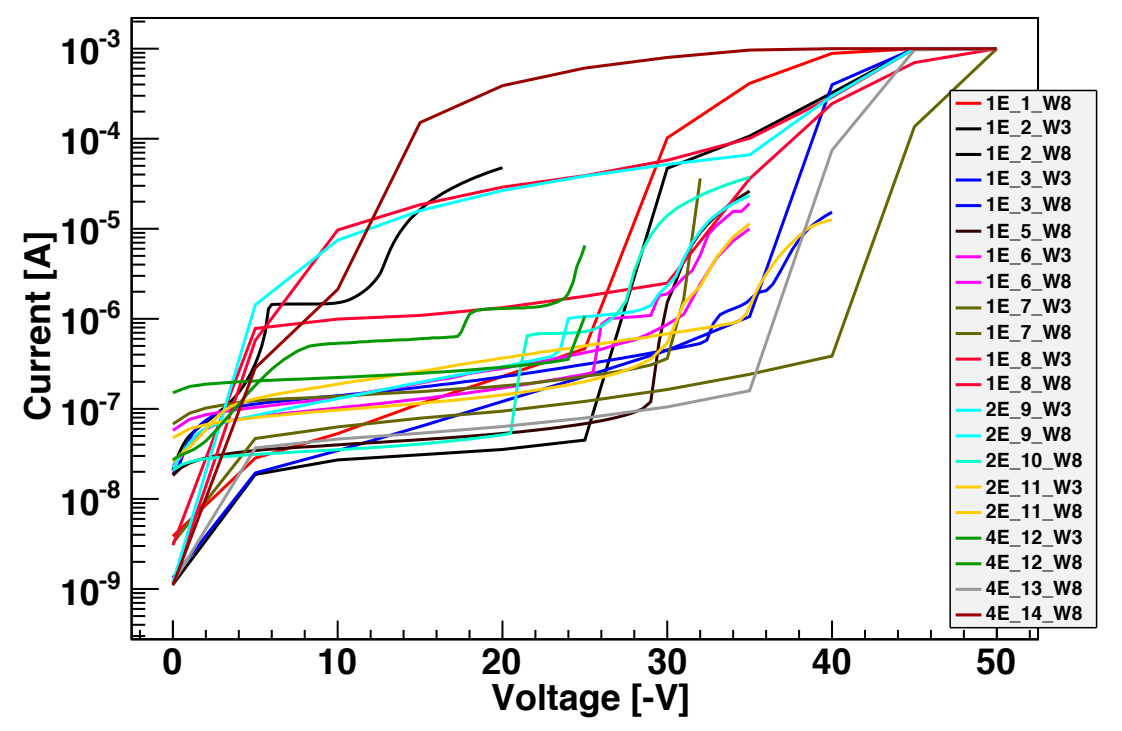

Figure 12. Leakage currents as a function of reverse bias voltage. In large fraction of devices, breakdown occurs at $30 \mathrm{~V}$ or higher. High current and early breakdown are observed in few devices because of fabrication induced defects.

systematic fluctuations. Leakage current curves as a function of reverse bias voltage for 3D detectors considered in this study are shown in figure 12. Most of the investigated devices are working properly, showing leakage currents of less than $300 \mathrm{nA}$ and breakdown voltages above $20 \mathrm{~V}$. Although these breakdown voltage values appear very low, they are well understood and do not affect the charge collection efficiency since 3D devices are fully depleted at around $10 \mathrm{~V}$. The breakdown is attributed to the high p-spray implanted dose. The p-spray implanted dose was reduced in later batches and the reverse discharge was found to occur at higher voltages [17]. There are few devices that exhibit much higher currents and/or premature breakdown. These effects are most likely caused by fabrication induced defects inside the sensors. The overall sensor breakdown voltages are given in table 1 . From this table, it can be concluded that $62 \%$ of the sensors compare well with simulation results. It should be stressed that no preliminary electrical tests were performed on these pixel sensors at wafer level. In later batches processed at FBK, leakage currents are monitored on all sensors by using a temporary metal layer, in order to exclude bad sensors from being bump bonded [24].

\subsection{Noise}

An S-curve test is used to determine the pixel noise. The S-curve is obtained by sending internal calibration signals through the injection capacitor to the ROC preamplifier input and measuring the sensor pixel response efficiency on a pixel-by-pixel basis. Then an error function is fitted to each pixel S-curve. The pixel noise is determined from the curve at the turning point. A typical S-curve plot for one pixel and the noise distribution of all pixels in a 1E sensor are shown in figure 13. The overall noise level is determined by fitting the noise distribution with a Gaussian function (shown as a dashed red line). The determined noise level is 208 electrons with an RMS spread of 18 electrons. The sensors have edge pixels which have larger dimensions than the nominal pixel size of 100 $\times 150 \mu \mathrm{m}^{2}$. Therefore, these pixels are noisier due to their larger capacitance. The small bump be- 
Table 1. Measured breakdown voltages for all the sensors.

\begin{tabular}{|ccc|}
\hline Number of sensors & Breakdown voltage [-V] & Fraction [\%] \\
\hline 4 & between 10 and 15 & 19 \\
4 & 25 & 19 \\
13 & between 30 and 40 & 62 \\
\hline
\end{tabular}
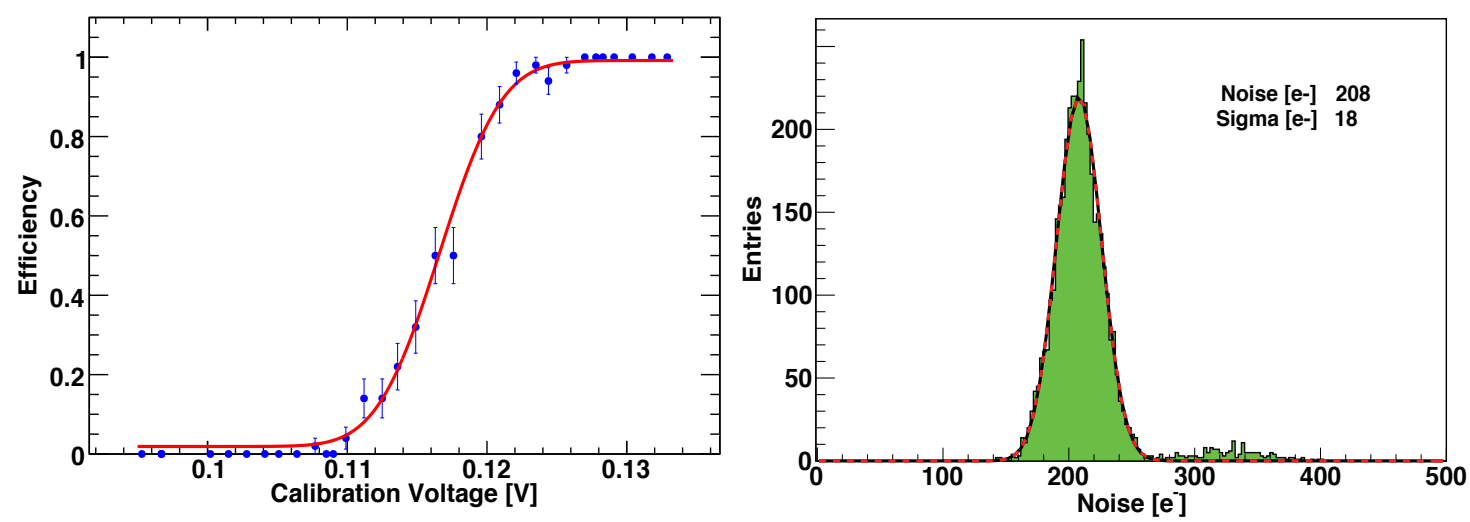

Figure 13. (Left) S-curve fit with an error function for quantifying the noise level of a pixel. (Right) Noise

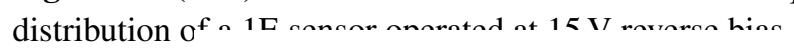

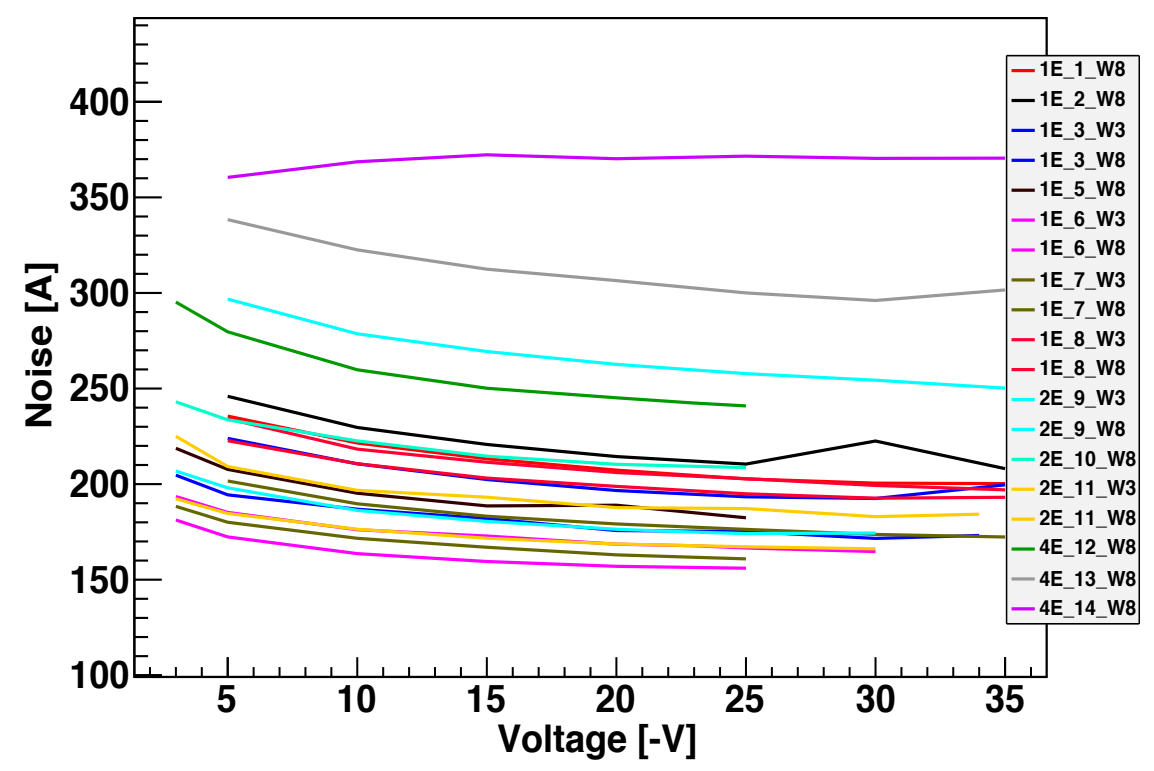

Figure 14. Measured noise as a function of reverse bias voltage. In all devices, the full depletion is observed at $10 \mathrm{~V}$. Noise changes with the number of electrodes. The higher the number of electrodes in one pixel, the higher the capacitance and the higher the noise.

tween 300 and 400 electrons in the noise distribution represents the noise of the larger edge pixels.

The sensor noise decreases as the reverse bias increases and reaches a plateau at a certain bias 
voltage, as seen in figure 14. The plateau indicates full sensor depletion. This decrease in noise is due to the decrease in pixel capacitances until the sensor is fully depleted. The reverse bias at which the full depletion occurs is taken as the operational voltage. In all examined 3D sensors, the measured full depletion voltage is approximately $10 \mathrm{~V}$. Average sensor noise at full depletion is 230 electrons for $1 \mathrm{E}$ sensors, 280 electrons for $2 \mathrm{E}$ sensors, and 330 electrons for $4 \mathrm{E}$ sensors. The noise is higher than the 155 electrons observed in planar FPIX sensors currently operational in the CMS experiment at CERN [25]. This is expected because of the larger capacitance of 3D sensors with respect to planar sensors.

\subsection{Charge collection}

$\mathrm{A}{ }^{90} \mathrm{Sr}$ radioactive source is used for the sensor charge-collection studies. The source is placed in close proximity to the sensor. Because of the very high activity of the source, 5 seconds of data taking is sufficient to collect 100,000 events. A trimmed readout threshold of 3,900 electrons is applied to all the sensors during data taking. Pixel cells with charge above the readout threshold are read out. Pixel clusters are formed by grouping adjacent pixels. Both neighboring side and corner pixels are included in the cluster. The cluster charge in ADC counts is converted into electrons using the pulse-height calibration, which correlates ADC counts with the ionization charge. Cluster charges are first converted from ADC to internal calibration voltage (Vcal) unit, which corresponds to 65.5 electrons, then charges in Vcal units are converted to electrons.

Electrons from radioactive source release their energy in silicon by ionization energy loss, which is approximated by a Landau distribution. However, because of the Gaussian distribution of the electronics noise, the convolution of a Landau and a Gaussian distribution is used to fit the data. The total cluster-charge distribution of a $4 \mathrm{E}$ sensor measured at $15 \mathrm{~V}$ reverse bias is shown in figure 15 in Vcal units. The dashed red line is the result of the fit. The collected charge is defined as the most probable value (MPV) of a Landau convoluted with a Gaussian function, fitted to the total cluster charge distribution. The MPV value is about $14 \mathrm{ke}$. The expected number of electrons from a $200 \mu \mathrm{m}$ thick silicon is $15 \mathrm{ke}$, assuming the most probable value of $\sim 75$ electron-hole pairs that are generated per micron of silicon at room temperature [26].

The charge-collection is also measured as a function of reverse bias voltage, as shown in figure 16, to determine the sensor charge-collection efficiency and to provide an optimum setting for each sensor. The points on the figure are the measured Landau MPV values. Sensor chargecollection increases for increasing bias voltages until it reaches a plateau. Saturation begins at $10 \mathrm{~V}$ reverse bias for most of the sensors, regardless of their electrode configuration, in good agreement with the full depletion voltage extracted from the $\mathrm{C}-\mathrm{V}$ curves. The collected charge values are not exactly the same for all sensors, although the substrate thickness is same: this is ascribed to varying ROC-to-ROC pulse height calibrations.

The signal-to-noise $(\mathrm{S} / \mathrm{N})$ ratio for all sensors is quantified by using measured Landau MPV values as signal and Gaussian mean value of noise distributions as noise. Average measured S/N ratios for sensors at full depletion are shown in table 2 . 


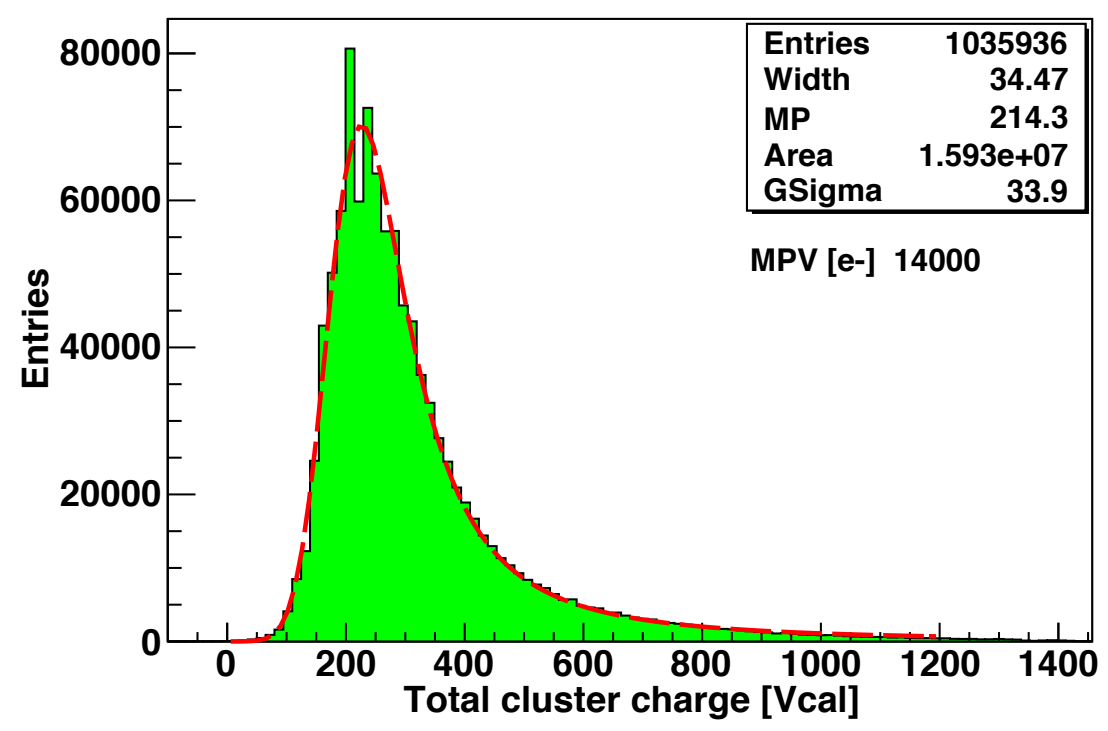

Figure 15. Total cluster charge for the $4 \mathrm{E}$ sensor operated at reverse bias of $15 \mathrm{~V}$. The dashed red line is the result of the fit.

Table 2. Average measured $\mathrm{S} / \mathrm{N}$ ratios for all the sensors at full depletion.

\begin{tabular}{|cc|}
\hline Electrode configuration & $\mathrm{S} / \mathrm{N}$ \\
\hline $1 \mathrm{E}$ & 66 \\
$2 \mathrm{E}$ & 46 \\
$4 \mathrm{E}$ & 36 \\
\hline
\end{tabular}

\section{Comparison of simulation results with laboratory measurements}

\subsection{Leakage current}

Simulated currents are in good agreement with measured values, at least for those devices that do not exhibit early breakdown or very high leakage currents (see table 3). Comparing current values at an operating voltage of $20 \mathrm{~V}$, simulated currents are slightly lower than the measured ones, as shown in figure 17. This difference can be attributed to the following effects: (i) in real devices, edge pixels are larger and therefore draw a higher current, a fact is not taken into account in the simulation which focuses on the central pixels, (ii) the plateau slopes of the measured currents as a function of voltage are higher than those of the simulated ones, and this difference is attributed to surface effects (i.e. energetic distribution of the interface states), which are difficult to properly model. Good agreement was also found for the breakdown voltages. Simulated and measured values are comparable within a $\sim 5 \mathrm{~V}$ margin, excluding from the comparison the devices with an abrupt current increase at voltages lower than $20 \mathrm{~V}$, as shown in table 3 . A few devices have higher breakdown voltage $(\sim 40 \mathrm{~V})$ and this is probably due to different amounts of oxide charge concentration. It is known that in p-spray isolated structures a higher charge concentration can increase the breakdown voltage [27]. In the considered batch (ATLAS08) the oxide charge concentration 


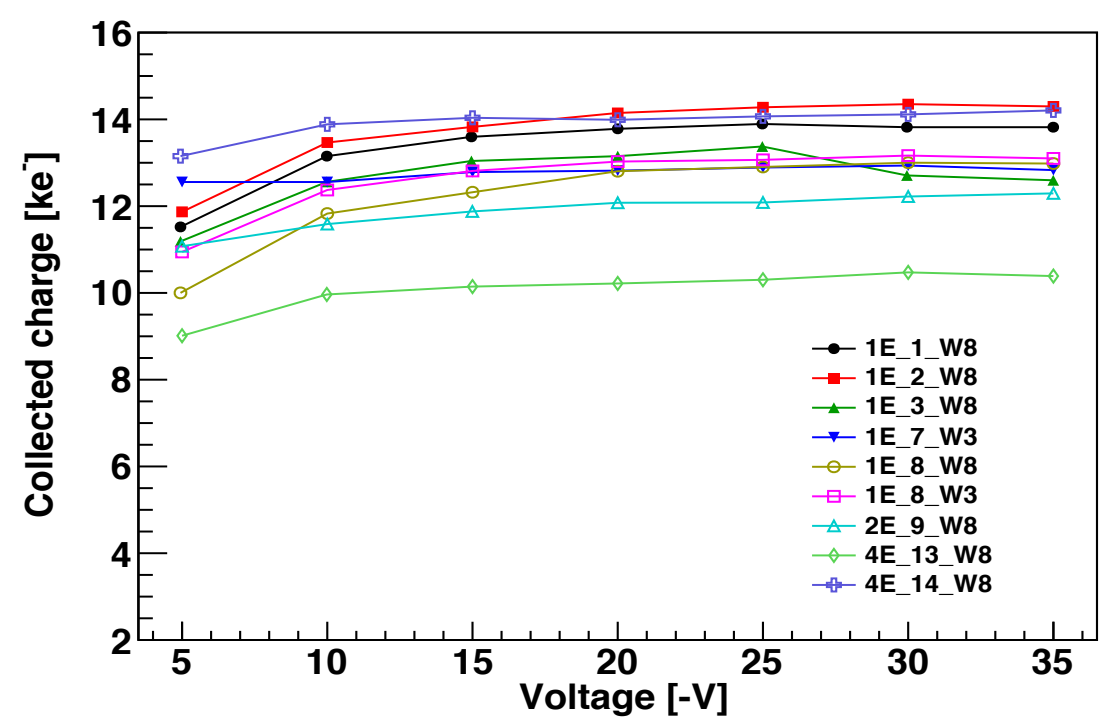

Figure 16. Measured collected charge as a function of reverse bias voltage. Each measured point is the most probable value of a Landau convoluted with a Gaussian function fit.

Table 3. Simulated and measured leakage currents as a function of reverse bias voltage for all the sensors.

\begin{tabular}{|ccc|}
\hline Electrode configuration & Breakdown voltage [-V] & Leakage current [nA] \\
\hline & Simulated - Measured & Simulated - Measured \\
\cline { 2 - 3 } 1E & $33-20$ to 40 & $42-31$ to 193 \\
2E & $31-20$ to 38 & $44-41$ to 264 \\
4E & $30-20$ to 35 & $52-53$ to 248 \\
\hline
\end{tabular}

was indeed found to be non-uniform over the wafer surface (measured values range from 2 to $6 \times 10^{11} \mathrm{~cm}^{-2}$ ), so that fluctuations of the breakdown voltage of up to a few volts are expected.

\subsection{Charge collection}

Simulated and measured charge collection results are in good agreement except that the maximum values of the collected charge are lower than the simulated value of $16 \mathrm{ke}$ - (figure 18). We attribute this difference to few concurring factors. The first factor is charge sharing. Further data analysis showed that electrons mostly arrived to sensors at a non-zero angle because the radioactive source, which has a $50 \mu \mathrm{m}$ opening window, was placed $1 \mathrm{~cm}$ from the sensor surface. No collimator was used. It was observed from hit occupancy plot that more than $90 \%$ of the sensor area was covered. The beam angle of incidence was determined to be 80 degrees, enhancing the charge sharing that was confirmed by event cluster size distributions. Charge collection is maximum when the beam angle of incidence is zero. At non-zero angle of incidence, charge sharing increases and a certain fraction of charge is not measured in adjacent pixels that do not go over the readout threshold of 3,900 electrons. Therefore, collected charge decreases [28, 29]. The second factor is the uncertainty of sensor thickness that can vary few tens of microns (see section 2). As a matter of fact, from $\mathrm{C}-\mathrm{V}$ curves of planar test diodes a thickness of about $185 \mu \mathrm{m}$ was extracted. The third 


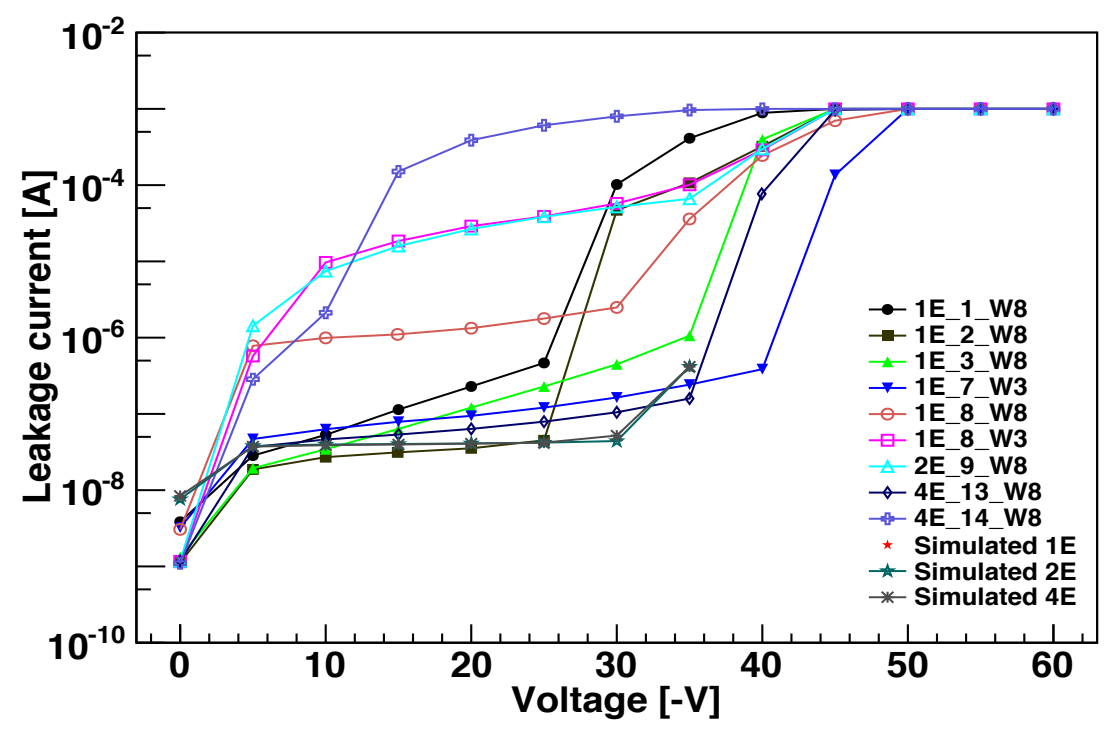

Figure 17. Simulated and measured leakage currents as a function of reverse bias voltage. Devices with lower breakdown are excluded. The plateau slopes of the measured curves are higher than simulated ones due to surface effects. A $5 \mathrm{~V}$ margin of breakdown voltage is observed between simulated and measured curves.

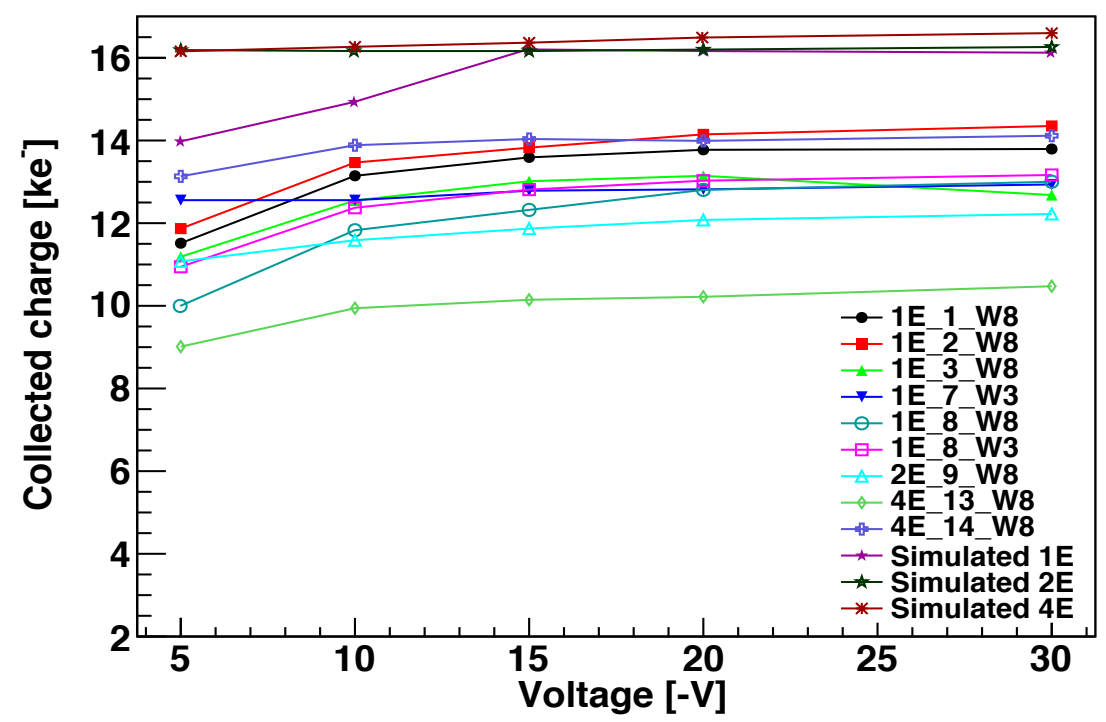

Figure 18. Simulated and measured collected charge as a function of reverse bias voltage. Simulated charge values are higher than measured ones. Concurring factors responsible for this discrepancy are explained in the text.

factor is the uncertainty of the calibration of the Analog-to-Digital-Converter(ADC) units of the DAQ system in terms of number of electrons.

\subsection{Noise}

A study has been performed to determine the relationship between measured pixel noise and simulated capacitance, under the assumption that the noise of a bare ROC is constant. The bare ROC noise of 100 electrons was measured with the S-curve test (see section 5.2), by means of the same 
DAQ system used for 3D detector performance tests. A nearly linear relationship between measured pixel noise and simulated capacitance is observed for all sensor types. The linear behavior suggests that simulated capacitance are in good agreement with the measured noises. It also suggests that ROC noise is fairly constant, independent of sensor type, and can therefore be ignored.

\section{Conclusions}

The 3D CMS pixel sensors considered in this study have ohmic and junction electrodes, which are etched from opposite surfaces. Both electrode types completely pass through the silicon bulk and are hollow. These sensors were designed at the University of Trento and fabricated at FBK in Italy. They were diced and bump bonded to the CMS pixel PSI46v2 readout chips at SELEX in Italy. Module assembly and lab characterization measurements were performed at Purdue P3MD and INFN Torino laboratories. Most of the assembled devices are working properly. A thorough simulation was performed to better understand the sensor behavior and to confirm laboratory results. All laboratory measurements were performed at room temperature. The leakage currents of good sensors are below $300 \mathrm{nA}$ and breakdown voltages between $30 \mathrm{~V}$ and $40 \mathrm{~V}$. Noise characteristics of these sensors are higher compared to conventional planar sensors due to larger pixel capacitances as a consequence of shorter inter-electrode distances. Average sensor noise at full depletion was measured 230 electrons for $1 \mathrm{E}$ configuration, 280 electrons for $2 \mathrm{E}$ configuration, and 330 electrons for $4 \mathrm{E}$ configuration. Charge-collection study was performed by using a radioactive source. The average MPV value was measured to be $14 \mathrm{ke}$ - in contrast to simulated $16 \mathrm{ke}$-. Laboratory and simulation results have shown a good agreement in the electrical parameters, whereas some discrepancies were observed in the charge collection. Although these discrepancies are mostly understood, they will still be further investigated.

3D technologies are a good candidate for the CMS pixel upgrade for HL-LHC. They are expected to outperform planar sensors in very harsh radiation environments. Sensor post-irradiation studies are under way. Sensors were irradiated with $800 \mathrm{MeV}$ protons in December 2011 at the Los Alamos National Laboratory, U.S.A. Post-irradiation sensor characterization will be carried out both in laboratory and test beams. Post-irradiation investigations will provide more hints to evaluate radiation hardness of 3D sensors.

\section{Acknowledgments}

This work was supported in part by the U.S. Department of Energy under Grant DE-FG0291ER40681, in part by the National Science Foundation under Cooperative Agreement PHY 0612805 UCLA Subaward 1000 G HD 870, in part by the Provincia Autonoma di Trento through the Project MEMS2, and in part by the Italian National Institute for Nuclear Physics (INFN) through the CSN5 Project TREDI.

The PSI46v2 ROCs, which were used in this study, were designed and developed by R. Horisberger's research group (W. Erdmann, R. Horisberger, H.C. Kästli, and B. Meier) at Paul Scherrer Institute in Switzerland. The authors would like to thank to Mark Smith at Purdue University for his tremendous help, would like to express their appreciation to US CMS PIXEL PIRE members, especially Dr. Jose Lazo-Flores, for their assistance to install and debug the DAQ system in the 
P3MD lab. The support of Simonetta Di Gioia (Selex SI) for the bump-bonding process and of Barbara Pini, Florea Dumitrache (INFN Torino), and Gale Lockwood (Purdue University) for the wire-bonding process is also acknowledged. We thank Prof. Michele Arneodo for his careful reading of the manuscript.

\section{References}

[1] CMS collaboration, G. Auzinger, Silicon sensor development for the CMS tracker upgrade, 2011 JINST 6 P10010 [INSPIRE].

[2] T. Rohe et al., Radiation hardness of CMS pixel barrel modules, Nucl. Instrum. Meth. A 624 (2010) 414 [arXiv: 1001.0666 ] [inSPIRE].

[3] G. Cerati et al., Radiation tolerance of the CMS forward pixel detector, Nucl. Instrum. Meth. A 6000 (2009) 408 [INSPIRE].

[4] FP420 R AND D collaboration, M. Albrow et al., The FP420 R \& D Project: Higgs and New Physics with forward protons at the LHC, 2009 JINST 4 T10001 [arXiv: 0806 . 0302] [INSPIRE].

[5] S.I. Parker, C.J. Kenney and J. Segal, 3D: A New architecture for solid state radiation detectors, Nucl. Instrum. Meth. A 395 (1997) 328 [INSPIRE].

[6] C. Kenney et al., Silicon detectors with 3-D electrode arrays: fabrication and initial test results, IEEE Trans. Nucl. Sci. 46 (1999) 1224.

[7] T.-E. Hansen et al., First fabrication of full 3D-detectors at SINTEF, 2009 JINST 4 P03010 [iNSPIRE].

[8] O. Koybasi et al., Electrical Characterization and Preliminary Beam Test Results of 3D Silicon CMS Pixel Detectors, IEEE Trans. Nucl. Sci. 58 (2011) 1315.

[9] A. Zoboli et al., Double-sided, Double-Type Column 3-D Detectors: Design, Fabrication and Technology Evaluation, IEEE Trans. Nucl. Sci. 55 (2008) 2775.

[10] G. Pellegrini et al., First double-sided 3-D detectors fabricated at CNM-IMB, Nucl. Instrum. Meth. A 592 (2008) 38 [INSPIRE].

[11] C.J. Kenney et al., Results from 3-D silicon sensors with wall electrodes: near-cell-edge sensitivity measurements as a preview of active-edge sensors, IEEE Trans. Nucl. Sci. 48 (2001) 2405.

[12] C. Da Via, J. Hasi, C. Kenney, A. Kok and S. Parker, 3D silicon detectors-status and applications, Nucl. Instrum. Meth. A 549 (2005) 122 [inSPIRE].

[13] A. Kok et al., 3D detectors-state of the art, Nucl. Instrum. Meth. A 560 (2006) 127.

[14] C.J. Kenney et al., Active-edge planar radiation sensors, Nucl. Instrum. Meth. Phys. A 565 (2006) 272.

[15] M.J. Bosma et al., Edgeless silicon sensors for Medipix-based large-area X-ray imaging detectors, 2011 JINST 6 C01035.

[16] G.-F. Dalla Betta et al., Development of modified 3D detectors at FBK, IEEE Nucl. Sci. Symp. Conf. Rec. 2010 (2010) 382.

[17] E. Vianello et al., Optimization of double side 3D detector technology for first production at FBK, IEEE Nucl. Sci. Symp. Conf. Rec. 2011 (2011) 523.

[18] M. Povoli et al., Slim edges in double-sided silicon 3D detectors, 2012 JINST 7 C01015 [INSPIRE]. 
[19] H.C. Kästli et al., Design and performance of the CMS pixel detector readout chip, Nucl. Instrum. Meth. A 565 (2006) 188 [physics/0511166] [inSPIRE].

[20] Synopsys Inc., Synopsys TCAD.

[21] M. Povoli et al., Impact of the layout on the electrical characteristics of double-sided silicon $3 D$ sensors fabricated at FBK, online version in Nucl. Instrum. Meth. A.

[22] O. Koybasi et al., Assembly and qualification procedures of CMS forward pixel detector modules, Nucl. Instrum. Meth. A 638 (2011) 55 [inSPIRE].

[23] A. Starodumov et al., Qualification procedures of the CMS pixel barrel modules, Nucl. Instrum. Meth. A 565 (2006) 67 [physics/0510165] [inSPIRE].

[24] C. Da Via et al., 3D active edge silicon sensors: Device processing, yield and QA for the ATLAS-IBL production, online version in Nucl. Instrum. Meth. A.

[25] A. Kumar et al., Commissioning of the CMS forward pixel detector, 2009 JINST 403026.

[26] H. Bichsel, Straggling in thin silicon detectors, Rev. Mod. Phys. 60 (1988) 663 [INSPIRE].

[27] C. Piemonte, Device simulations of isolation techniques for silicon microstrip detectors made on p-type substrates, IEEE Trans. Nucl. Sci. 53 (2006) 1694.

[28] M. Mathes et al., Test Beam Characterization of 3-D Silicon Pixel Detectors, IEEE Trans. Nucl. Sci. 55 (2008) 3731.

[29] P. Grenier et al., Testbeam results of $3 D$ silicon pixel sensors for the ATLAS upgrade, Nucl. Instrum. Meth. A 638 (2011) 33. 